\title{
Rosazea und Mevalonazidurie bei einem 11-jährigen Kind
}

\section{Rosacea and Mevalonic Aciduria in an 11-Year-Old Child}

Autoren

Institut
A. Kasche-Segnitz, V. Mielke, U. Reusch, M. Reusch, E. Christophers

Fachübergreifende Gemeinschaftspraxis für Dermatologie und Pathologie, Hamburg

\section{Bibliografie}

DOI 10.1055/s-2007-966310

Akt Dermatol 2007; 33 :

76-78 @ Georg Thieme

Verlag KG Stuttgart · New York ISSN 0340-2541

\section{Korrespondenzadresse}

Dr. med. Anna Kasche-Segnitz

Fachübergreifende Gemeinschaftspraxis für Dermatologie und Pathologie

Tibarg 7-9 22459 Hamburg

Hautaerzte-am-Tibarg@

t-online.de

\section{Zusammenfassung}

$\nabla$

Bei einem 11-jährigen Jungen mit einer Mevalonazidurie kam es nach systemischer Glukokortikosteroidtherapie zum Auftreten einer Rosazea.

\section{Einleitung}

$\nabla$

Mevalonazidurie (MA) ist ein seltenes, kürzlich beschriebenes Krankheitsbild, das bis heute bei insgesamt etwa 30 Fällen beobachtet wurde [1]. Die Ursache ist ein genetisch bedingter Mangel an Melavonatkinase (MVK). Dieses Enzym reguliert die Biosynthese von Cholesterin und nichtsteroidalen Isoprenoiden [2]. Die Krankheitssymptome beruhen auf einem Mangel der Endprodukte der MVK und einer gestörten Regulation des verzweigten Biosyntheseweges. Ein defektes Gen wurde auf Chromosom 12 (12q24) beschrieben [3].

Das klinische Bild dieser Enzym-Mangelerkrankung ist vielgestaltig und spiegelt sich in einer großen Zahl von Einzeldefekten und Symptomen wider: allen voran frühe Wachstums- und Gedeihstörungen und neurologische Symptome (zerebelläre Ataxie). Im weiteren Verlauf zeigen sich Katarakte, retinale Dystrophien, Hepatosplenomegalie und Arthralgien. Ein weiteres Charakteristikum sind plötzlich einsetzende Fieberschübe mit Diarrhöen und ausgeprägter Lymphknotenschwellung [4].

Diagnostisches Kriterium sind exzessive Mevalonsäure-Mengen im Urin, Anämie, Kreatininkinase- und Transaminase-Erhöhungen $[2,4]$.

Wir beobachteten einen 11-jährigen Jungen, bei dem im ersten Lebensjahr eine MA erkannt wurde und der nachfolgend eine Rosazea entwickelte.
Unter topischer Therapie mit Metronidazol und Erythromycin sowie Reduktion der Steroiddosis zeigte sich eine deutliche Besserung des Hautbefundes.

\section{Kasuistik \\ $\nabla$}

Anamnese und Verlauf

Der Patient ist das erste Kind gesunder, nicht verwandter Eltern. Er hat einen älteren, gesunden Halbbruder. Die Mutter kommt aus Süditalien und der Vater aus Kroatien.

Auffällig wurde der Patient in der 6. Lebensstunde, als an der Haut blau-graue, makulöse gesichtund stammbetonte Effloreszenzen auftraten. Sie wurden im Verlauf der Erkrankung als Zeichen einer extramedullären Hämatopoese erkannt. Im Verlauf entwickelten sich eine Hepatosplenomegalie und Anämie sowie ansteigende Werte für das direkte und indirekte Bilirubin. Die Lebertransaminasen sowie die Laktatdehydrogenase waren leicht erhöht [5].

Im Urin wurde ein Peak im Bereich der Mevalonsäure gemessen und es wurde in der Kinderklinik des Universitätsklinikums Hamburg-Eppendorf in Zusammenarbeit mit Prof. G. F. Hoffmann die Diagnose einer Mevalonazidurie gestellt. Bei dem Jungen kam es nachfolgend alle 4 bis 8 Wochen zum Auftreten von Fieberschüben.

Die ophthalmologische Untersuchung ergab eine Hyperopie, Astigmatismus sowie Amblyopie links.

Im Alter von 11 Jahren wiegt der Junge nur $19 \mathrm{~kg}$ und ist nur $102 \mathrm{~cm}$ groß, weist eine zerebelläre Ataxie mit Gangstörungen und eine psychomotorische Retardierung auf. 


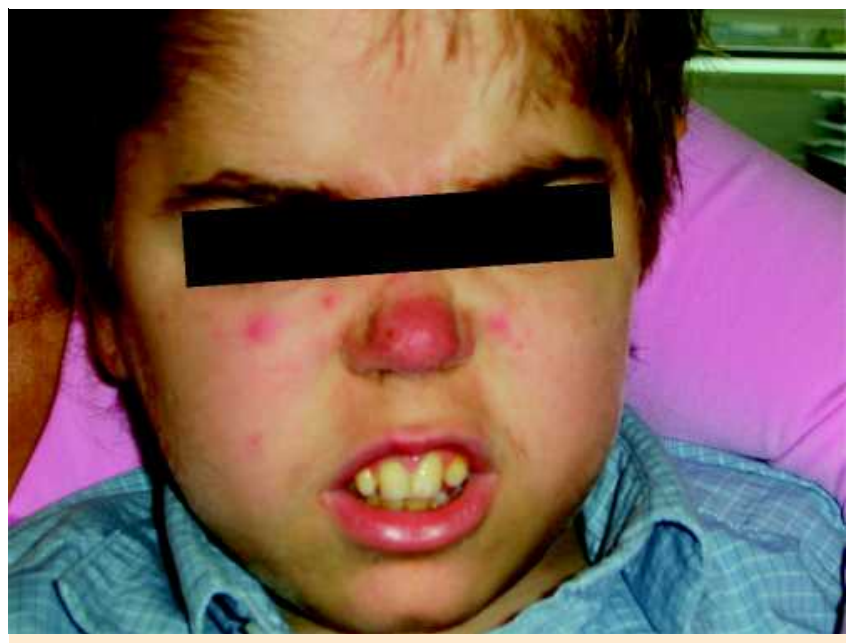

Abb. 1 Vor Therapie: Klinisches Bild einer Rosazea.

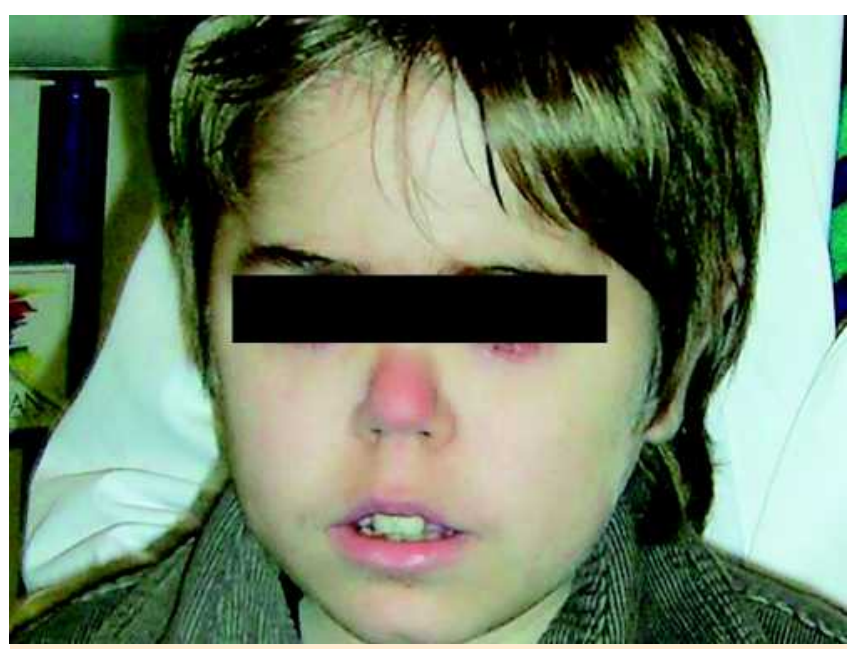

Abb. 2 Nach Therapie: Deutliche Besserung des Hautbefundes nach topischer Therapie mit Metronidazol und Erythromycin sowie Reduktion der oralen Glukokortikosteroiddosis.

\section{Aufnahmebefund}

Follikulär konfluierende, münzgroße und hochrote Verfärbung der Nase, die scharf begrenzt ist und von einzelnen Pusteln durchsetzt wird ( $\odot$ Abb. 1). Die Umgebung erscheint nicht verändert. Morphologisch gleiche Herde finden sich im Bereiche beider Ohren am Meatus externus.

Schwellung der nuchalen Lymphknoten mit linksseitiger Betonung.

\section{Befunde diagnostischer Untersuchungen}

(Labor Prof. Arndt \& Partner; Medizinisches Labor Bremen, Prof. Kühn-Velten)

\section{Mevalonsäure im Urin}

Die Bestimmung von Mevalonolacton (Ringform der Mevalonsäure) lieferte ein sehr intensives Signal für diese Substanz. Der Messwert zeigte eine massive Erhöhung oberhalb des Kalibrationsbereiches von $2 \mathrm{mg} / \mathrm{l}$ (entsprechend $15 \mathrm{umol} / \mathrm{l}$ ).

Immunglobulin D:

$36,0 \mathrm{IU} / \mathrm{ml}$ (Norm 2,0-100,0).
Molekulargenetische Diagnostik:

Die molekulargenetische Untersuchung von Leukozyten ergab Veränderungen auf Chromosom 12. Es liegt ein Compound-Heterozygotenstatus für die Mutation T237S und I268T im Mevalonatkinase-Gen vor. Diese beiden Mutationen sind in der Literatur beschrieben $[6,7]$.

\section{Therapie und Verlauf}

Der kleinwüchsige 11-jährige Patient erhielt mit Beginn im ersten Lebensjahr Prednison $5 \mathrm{mg} / \mathrm{die}$. Jeweils bei Dosisreduktion kam es zu einer deutlichen Verschlechterung und Verstärkung der Symptome. Daher wurde die Dosis $5 \mathrm{mg} /$ die über mehr als 10 Jahre beibehalten. Wegen rezidivierend auftretender anämischer Schübe wurden zeitweilig Bluttransfusionen verabfolgt. Unter dieser Behandlung (Prednison $5 \mathrm{mg} /$ die) entwickelten sich erythematöse Bezirke an Nase und Ohren und das Bild einer Rosazea.

Unter Reduktion der Prednison-Dosis auf $2,5 \mathrm{mg} / \mathrm{die}$ und topischer Therapie mit Metronidazol 0,5 Linola Emulsion ad 50,0 g und Ichthyol 0.6 Sulfur praecip 0.6 Pasta zinci mollis ad 30,0 g sowie Erythromycin 0.36 Anefug simplex ad $16.0 \mathrm{~g}$ zeigte sich eine deutliche Besserung der Rosazea.

\section{Diskussion}

Das defekte Mevalonatkinase (MVK) Gen auf Chromosom 12 ist ursächlich für zwei Syndrome verantwortlich: die Mevalonazidurie (MA) und das Hyper-IgD-und-periodisches-Fiebersyndrom (HIDS). Sie werden autosomal rezessiv vererbt und zeigen sich in klinisch sehr unterschiedlichen Krankheitsformen:

Bei der kompletten Defizienz der Enzymaktivität kommt es im frühen Kindesalter zur Ausprägung einer MA, einer schweren, oft letal verlaufenden Multisystemerkrankung. Charakteristische Merkmale für die MA sind psychomotorische Retardierung, Gedeihstörungen, Lymphadenopathie, Hepatosplenomegalie, Anämie und periodische Fieberattacken [2,4].

Im Unterschied zur Mevalonazidurie geht das Hyper-IgD-undperiodisches-Fiebersyndrom (HIDS) mit einem partiellen Verlust der Mevalonatkinase-Aktivität einher [6]. Die Patienten leiden an rezidivierenden, plötzlich einsetzenden Fieberschüben von drei bis sieben Tagen Dauer, die sich etwa alle vier bis acht Wochen wiederholen. Die Fieberattacken werden häufig begleitet von gastrointestinalen Funktionsstörungen (Bauchschmerzen, Durchfall, Erbrechen), Lymphadenopathie, makulösen Exanthemen, Arthritiden an großen Gelenken und Polyarthralgien [8], nicht aber von gravierenden neurologischen Veränderungen und Wachstumsstörungen.

Diagnostische Labormarker für HIDS (im Gegensatz zur MA) sind eine Erhöhung des Immunglobulin D im Serum auf mehr als $100 \mathrm{IU} / \mathrm{ml}$ sowie eine erhöhte Ausscheidung von Mevalonsäure während der Fieberepisoden.

Im vorliegenden Fall konnte ein Compound-Heterozygotenstatus für die Mutation T237S und I268T im Mevalonatkinase-Gen nachgewiesen werden. Beide Mutationen sind in der Literatur schon beschrieben worden [6,7].

Von der molekulargenetischen Untersuchung her kann unser Befund sowohl dem HIDS als auch der MA zugeordnet werden. Das klinische Bild bei unserem Patienten mit schweren Gedeihstörungen (geistige Retardierung und Minderwuchs) und neurologischen Störungen (zerebelläre Ataxie) entspricht jedoch eindeutig einer MA. Auch zeigten sich keine erhöhten Immunglobu- 
lin-D-Spiegel, ein Befund, der gegen das Vorliegen eines HIDS spricht.

Hautveränderungen werden beim HIDS am häufigsten als makulöse Exantheme beschrieben [8]. Ihre genaue diagnostische $\mathrm{Zu}-$ ordnung erscheint aufgrund der vorliegenden Literatur schwierig, wohl auch, da die gefächerte und pathogenetisch bislang nicht definierbare Symptomatik der Grunderkrankung im Vordergrund steht.

Die jetzt beobachteten Veränderungen an Nase und Ohren entsprechen klinisch dem Bild einer Rosazea. Nach anamnestischen Angaben der Mutter entstanden sie nach dem ersten Lebensjahr. Die Zuordnung dieser kutanen Symptomatik zum Krankheitsbild der HIDS erscheint ungewöhnlich und zwingt zum Nachdenken über weitere Ursachen.

Bei der MA führt die orale Verabreichung von Prednison zu rasch erfolgender Rückbildung der interkurrenten systemischen Symptomatik, vor allem der Fieberschübe und LymphknotenSchwellungen. So auch bei unserem kleinen Patienten. Die Glukokortikosteroidmedikation erfolgte deshalb über länger als ein Jahrzehnt und betrug $5 \mathrm{mg} /$ Tag. Versuche, die Therapie zu unterbrechen, wurden (von der Mutter) als Rezidiv-induzierend angesehen. Wohl aus diesem Grunde (einer langfristig erhöhten Glukokortikosteroidzufuhr) ist die Rosazea als klinische Folge der Therapie zu verstehen. Auch zeigten sich unsere Ansätze einer gezielten topischen Rosazea-Therapie als erfolgreich ( $\bullet$ Abb. 2). Im Laufe der Jahre kam es auch zu einem Rückgang der Fieberepisoden, sodass die Prednison-Dosis zuletzt auf $2,5 \mathrm{mg} / \mathrm{die}$ reduziert werden konnte.

\section{Abstract}

\section{Rosacea and Mevalonic Aciduria in an 11-Year-Old Child}

$\nabla$

We report on an 11-year-old boy with mevalonic aciduria who developed a rosacea after oral treatment with corticosteroids. After topical treatment with metronidazole and erythromycin and reduction of the steroid dosage we noted an obvious improvement of the skin lesions.

\section{Literatur}

1 Haas D, Hoffmann GF. Mevalonate kinase deficiences: from mevalonic aciduria to hyperimmunoglobulinemia D syndrome. Orphanet J Rare Dis 2006; $1: 13$

2 Hoffmann G, Gibson KM, Brandt IK, Bader PI, Wappner RS, Sweetman L. Mevalonic acid: an inborn error of cholesterol and nonsterol isoprene biosynthesis. N Engl J Med 1986; 314: 1610-1614

3 Gibson KM, Hoffmann GF, Tanaka RD, Bishop RW, Chambliss KL. Mevalonate kinase map position 12q24. Chromosome Research 1997; 5: 150

4 Hoffmann GF, Charpentier C, Mayatepek E, Mancini J, Leichsenring M, Gibson KM, Divry P, Hrebicek M, Lehnert W, Sartor K, Trefz FK, Rating $D$, Bremer HJ, Nyhan WL. Clincal and biochemical phenotype in 11 patients with mevalonic aciduria. Pediatrics 1993; 91: 915-921

5 Hinson DD, Rogers ZR, Hoffmann GF, Schachtele M, Fingerhut R, Kohlschutter A, Kelley RI, Gibson KM. Hematological abnormalities and cholestatic liver disease in two patients with mevalonate kinase deficiency. Am J Med Genet 1998; 78: 408-412

6 Houten SM, Kuis W, Duran M, de Koning TJ, van Royen-Kerkhof A, Romeijn GJ, Frenkel J, Dorland L, de Barse MMJ, Huijbers WAR, Rijkers GT, Waterham HR, Wanders RJA, Poll-The BT. Mutations in MVK, encoding mevalonate kinase, cause hyperimmunoglobulinaemia D and periodic fever syndrome. Nat Genet 1999; 22: 175-177

7 Mandey SH, Schneiders MS, Koster J, Waterham HR. Mutational spectrum and genotype-phenotype correlations in mevalonate kinase deficiency. Hum Mutat 2006; 27: 796-802

8 Drenth JP, Boom BW, Toonstra J, Van der Meer JW. Cutaneous manifestations and histologic findings in the hyperimmunoglobulinemia D syndrome. International Hyper IgD Study Group. Arch Dermatol 1994; 130: $59-65$ 